\title{
Real-Time DOP Ellipsoid in Polarization Mode Dispersion Monitoring System by Using PSO Algorithm
}

\author{
Xiaoguang Zhang ${ }^{1,2}$, Gaoyan Duan ${ }^{1,2}$, and Lixia $\mathrm{Xi}^{1,2}$ \\ ${ }^{1}$ Department of Physics, Beijing University of Posts and Telecommunications, Beijing \\ 100876, P.R. China \\ zhang.x.g@263.net \\ ${ }^{2}$ Key Laboratory of Optical Communication and Lightwave Technologies, Ministry of \\ Education, Beijing University of Posts and Telecommunications, P.R. China
}

\begin{abstract}
In high bit rate optical fiber communication systems, Polarization mode dispersion (PMD) is one of the main factors to signal distortion and needs to be compensated. PMD monitoring system is the key integral part of an adaptive PMD compensator. The degree of polarization (DOP) ellipsoid obtained by using a polarization scrambler can be used as a feedback signal for automatic PMD compensation. Generally, more than several thousands of sampling data of states of polarization (SOP) must be collected to insure getting a correct DOP ellipsoid. This would result in an unacceptable time consuming for adaptive PMD compensation. In this paper, we introduce the particle swarm optimization (PSO) algorithm in determining the real-time DOP ellipsoid with high precision, requiring only 100 sampling data of SOPs. Experimental results confirm that the PSO algorithm is effective for ellipsoid data fitting with high precision within $250 \mathrm{~ms}$ using our hardware environment.
\end{abstract}

Keywords: polarization mode dispersion, monitoring techniques, degree of polarization ellipsoid, particle swarm optimization algorithm.

\section{Introduction}

Polarization mode dispersion (PMD) is one of the main factors to signal distortion and one of the main limiting factors preventing capacity increase of optical fiber communication systems. PMD compensation has become one of hot topics in recent years [1-5]. An ordinary automatic PMD compensator can be divided into three important subparts: the PMD monitoring unit, the compensation unit and the control algorithm. An effective PMD monitoring system allows its monitoring signal to be highly correlated to PMD. An ideal PMD monitoring technique can reveal as much information as possible about the PMD vector, such as both the differential group delay (DGD), which is the magnitude of the vector, and the principal states of polarization (PSPs), the direction of the vector. The DOP ellipsoid obtained by using a polarization scrambler can determine both the DGD and PSP orientation by its three radii and the orientation angle of ellipsoid [2]. The polarization scrambler placed at the fiber input 
makes the input random states of polarization (SOPs) of light distribute uniformly over the whole Poincaré sphere in Stokes space. After propagation in a fiber link with PMD, all the output SOP points of light will constitute in Stokes space an ellipsoid (DOP ellipsoid) whose shape is determined by DGD and PSP of the fiber link. In order to extract the PMD information such as DGD or PSP, we need to get the analytical DOP ellipsoid equation from all these measured discrete points of output SOPs. The more points of the output SOPs we measure, the more accurate ellipsoid equation we get, and much time elapse. Generally, an ellipsoid can be distinguished after several thousands of SOPs points are measured, whereas the real-time adaptive PMD compensator requires a real-time feedback signal. In this paper we introduce the particle swarm optimization (PSO) as a powerful data fitting algorithm for getting a precise analytical DOP ellipsoid equation from only 100 measured SOP samples with fast speed, and getting real-time characteristic information of PMD such as DGD and PSP.

\section{A Brief Introduction to Polarization Mode Dispersion and PMD Compensation}

\subsection{Polarization Mode Dispersion}

Polarization mode dispersion has its origins in optical birefringence [3]. In a single mode fiber, an optical wave traveling in the fiber can be represented as the linear superposition of two orthogonal polarized $\mathrm{HE}_{11}$ modes. In an ideal fiber, with a perfect circular symmetric cross-section, the two modes $\mathrm{HE}_{11}^{x}$ and $\mathrm{HE}_{11}^{y}$ are indistinguishable (degenerate) with the same time group delay. However, real fibers have some amount of asymmetry due to imperfections in manufacturing process or mechanical stress on the fiber after manufacture as shown in Fig. 1. The asymmetry breaks the degeneracy of the $\mathrm{HE}_{11}^{x}$ and $\mathrm{HE}_{11}^{y}$ modes, resulting in birefringence with a difference in the phase and group velocities of two modes.
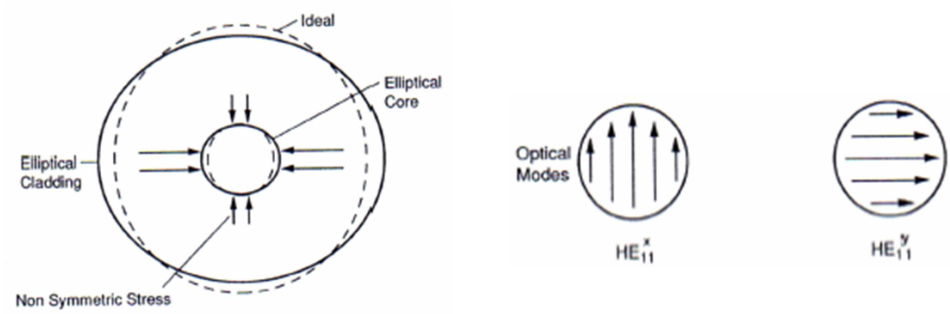

Fig. 1. Asymmetry of a real fiber and degeneracy of two orthogonal $\mathrm{HE}_{11}$ modes

If a pulsed optical wave that is linearly polarized at $45^{\circ}$ to the birefringence axis is launched into a birefringent fiber, the pulse will be splitted and separated at output end of the fiber due to the different group velocities between two $\mathrm{HE}_{11}$ modes, as shown in Fig. 2, resulting in a signal distortion in optical transmission system. The time separation between two modes is defined as differential group delay (DGD) $\Delta \tau$, which is the magnitude of PMD vector. Roughly speaking, the fast and slow axes are 
called principal states of polarization, one of which is defined as the direction of the PMD vector. This phenomenon is called polarization mode dispersion.

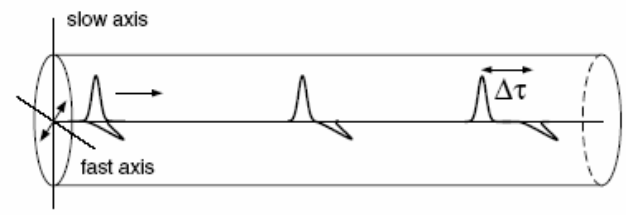

Fig. 2. Pulse splitting due to fiber birefringence

\subsection{The PMD Compensator and Monitoring Technique}

Fig. 3 shows a PMD compensation system. The optical signal is generated by the transmitter. The polarization scrambler placed just after the transmitter generates the random SOPs distributed over the whole Poincaré sphere in Stokes space. The optical signal will be distorted because of PMD in transmission fiber or made by PMD emulator. The control unit in PMD compensator makes the PMD compensation by adjusting the compensation unit according to the feedback signal generated by the PMD monitoring unit.

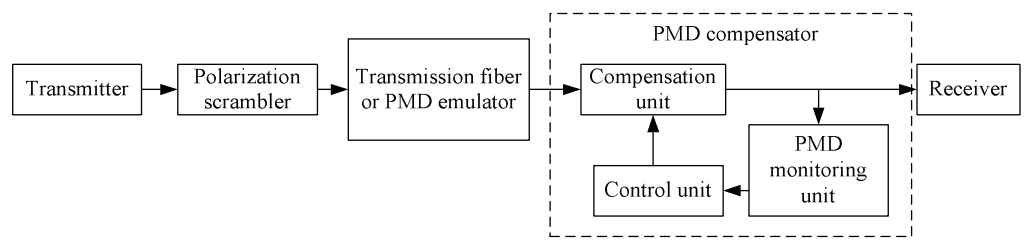

Fig. 3. The PMD compensation system

The PMD monitoring system is the key integral part of an adaptive PMD compensator. The degree of polarization (DOP) ellipsoid obtained by using a polarization scrambler can be used as a feedback monitoring signal for automatic PMD compensation.

\subsection{The Theory of DOP Ellipsoid}

DOP is defined by the four intensity related Stokes parameters $S_{0}, S_{1}, S_{2}, S_{3}$ according to the following equation:

$$
\mathrm{DOP}=\frac{\sqrt{S_{1}^{2}+S_{2}^{2}+S_{3}^{2}}}{S_{0}}
$$

The states of polarization (SOP) of the optical signals in the fiber link are represented by their Stokes parameters. The DOP ellipsoid obtained by using a polarization scrambler can determine both DGD and PSP orientation [5]. The 
polarization scrambler placed at the fiber input generates the random input SOP uniformly distributed over the whole Poincare sphere. If there is no PMD in the transmission fiber link, the Stokes parameters of the output signals satisfy the relation $S_{0}^{2}=S_{1}^{2}+S_{2}^{2}+S_{3}^{2}$, and hence DOP=1. All the output SOPs form a perfect ball sphere in Stoke space with unity radius. In the case of first-order PMD in the fiber link, the Stokes parameters satisfy the relation $S_{0}^{2}>S_{1}^{2}+S_{2}^{2}+S_{3}^{2}$, and DOP $<1$, except that when the input SOP is aligned with one of the PSPs (at this point DOP=1). So all the output SOPs form an ellipsoid whose longest radius has a length of unity and point into the direction of the PSPs.

For a system with only a first-order PMD, a DOP ellipsoid whose major axis is aligned with the PSP can be described as follows [5]

$$
S_{1}^{2}+\frac{S_{2}^{2}+S_{3}^{2}}{R^{2}(\tau)}=1
$$

where $\tau$ is the DGD and $R(\tau)$ is the normalized autocorrelation of the signal. Also its longest axis points along the $S_{1}$ axis. For general case, first-order PMD with PSP pointing in an arbitrary direction, have an ellipsoid with unity value for the longest radius ( $r_{\max }=1$ ) and the same value of $R(\tau)$ for the shortest radius ( $r_{\operatorname{mid}}=r_{\min }$ ), and with its longest axis oriented in the corresponding direction in Stokes space, is shown in Fig. 4.

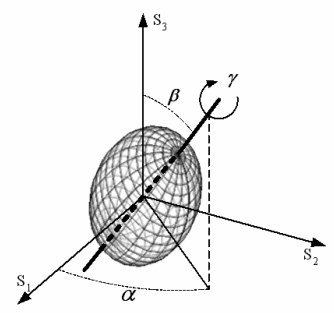

Fig. 4. The orientation of DOP ellipsoid in Stokes space

From the discussion above, we can see that the DOP ellipsoid is a good PMD monitoring signal, from which we can read out the detailed information related to PMD. Thus it can be used in feedback PMD compensation systems.

\section{PSO Technique Used to Obtain Real-Time DOP Ellipsoid}

The characteristic parameters for a given ellipsoid are its three radii and its orientation angles. We can get only the discrete sampling data of output SOPs from experiments. It is important to find a good algorithm to get the analytical ellipsoid equation from measured discrete data of output SOPs as fast as possible, in order to obtain instantly the parameters of the DOP ellipsoid such as the three radii of the ellipsoid $r_{\max }, r_{\text {mid }}$, $r_{\min }$ and its orientation angles $\alpha, \beta, \gamma$, as shown in Fig.4. The angles $\alpha$ and $\beta$ determine 
the direction of the longest axis, $\gamma$ determines the rotation angle around ellipsoid axis itself. This algorithm to be found is required to determine the correct ellipsoid with high precision using the fewest amounts of data, thus ensuring the speed requirement of PMD compensation to be satisfied.

We have previously introduced the particle swarm optimization (PSO) algorithm into automatic PMD compensation as a feedback control algorithm [4]. The PSO algorithm can be described as a procedure aimed at finding the global maximum or global minimum of a function in a multi-dimensional hyperspace by adjusting multicontrol parameters, shown mathematically as follows

$$
\underset{\text { parameters }}{\mathrm{MAX}}(\text { function })
$$

or

$$
\underset{\text { parameters }}{\operatorname{MIN}}(\text { function) }
$$

where the number of parameters is the number of dimensions of hyperspace.

In this paper we describe how, by introducing PSO technique into the PMD monitoring unit, we constructed an experiment for obtaining a real-time DOP ellipsoid by using the PSO algorithm in the form of expression (4).

A normal ellipsoid without tilt in the principal axis coordinate $S_{1}^{\prime \prime \prime}-S_{2}^{\prime \prime \prime}-S_{3}^{\prime \prime \prime}$ satisfies

$$
\frac{S_{1}^{\prime \prime \prime 2}}{r_{1}^{2}}+\frac{S_{2}^{\prime \prime 2}}{r_{2}^{2}}+\frac{S_{3}^{\prime \prime 2}}{r_{3}^{2}}=1
$$

In order to obtain a correct DOP ellipsoid, we firstly transform the measured sample data of Stokes parameters $S_{1 n}, S_{2 n}, S_{3 n}$ that constitute a tilt ellipsoid into the $S_{1 n}^{\prime \prime \prime}, S_{2 n}^{\prime \prime \prime}, S_{3 n}^{\prime \prime \prime}$ that are related to a normal ellipsoid in the principal axis coordinate, by three rotations $-\alpha,-\beta,-\gamma$. Secondly, we will continually adjust 6 parameters $\left(r_{1}, r_{2}\right.$, $\left.r_{3}, \alpha, \beta, \gamma\right)$ until following function in bracket is minimized:

$$
\operatorname{MIN}_{\left(r_{1}, r_{2}, r_{3}, \alpha, \beta, \gamma\right)}\left(\sum_{n=1}^{N}\left|\frac{S_{1 n}^{\prime \prime \prime 2}}{r_{1}^{2}}+\frac{S_{2 n}^{\prime \prime \prime 2}}{r_{2}^{2}}+\frac{S_{3 n}^{\prime \prime \prime 2}}{r_{3}^{2}}-1\right|\right)
$$

where $N$ is the number of sampling data points used for ellipsoid data fitting. Once the global minimum of the function in the bracket is found, we obtain the correct ellipsoid parameters $\left(r_{1}, r_{2}, r_{3}, \alpha, \beta, \gamma\right)$. This is a global minimum searching problem in the 6dimentional hyperspace. High number of degree of freedom will let us encounter the problem of being trapped in local sub-minima, which will result in the wrong ellipsoid. With the PSO algorithm, we can easily tackle the problem at a fast speed. The detail of the process of implementation of PSO can be found in reference [6].

\section{The Experiment and Results}

Fig. 5 shows the experimental setup for obtaining the DOP ellipsoid. The laser pulses with linear polarization state were generated by a fiber ring laser. The pulsewidth is 
7 -ps, and the central wavelength is $1560.5-\mathrm{nm}$. A polarization controller controlled by the computer was used as a polarization scrambler to randomly transform the SOPs of the laser signal to be uniformly distributed on entire Poincaré sphere, by randomly adjusting the three cells of the polarization controller. A computer controlled air gap time delay line was used as the first-order PMD emulator. A polarimeter detected the SOPs of the output optical signals, by getting the Stokes parameters $S_{0}, S_{1}, S_{2}, S_{3}$, and fed them into the computer through a 4-channel A/D. In the computer, the program with PSO algorithm performed the data fitting from sampled data, in order to obtain the DOP ellipsoid and hence the PMD information.

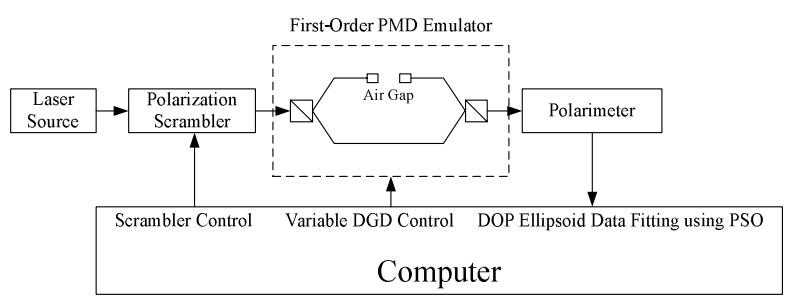

Fig. 5. Experiment scheme for DOP ellipsoid collection

Fig. 6 shows the 8000 sampling point graphs demonstrating the SOPs of the output optical signals obtained in our experiment, with various DGD values. These graphs just show the 8000 discrete output SOPs without any data fitting. It can be seen that, all the output SOPs form a ball sphere in Stokes space with zero DGD (Fig.6(a)), and a needle-like spheroid is formed with larger DGD (Fig.6(c)). The larger DGD is, the smaller value of the minimum radius of the ellipsoid is. Furthermore, according to the PMD theory, the PSPs of the PMD vector do not change for the first-order case. This accords with the results in Fig. 6(b) and Fig. 6(c) which show that the longest radius of ellipsoid remains oriented in the same direction with various DGDs.

Generally, the greater the size of the sampling data, the more accurate the data fitting ellipsoid is. But getting 8000 sampling data points combined with the 6dimentional adjusting parameter data fitting would be too time consuming using the hardware in our experiment (such as the scrambler, the 4-channel $\mathrm{A} / \mathrm{D}$, computer, etc), which is not acceptable for real-time PMD compensation. Just getting 8000 sampling points without any ellipsoid data fitting already consumed the time of 3815-ms in the experiment. In order to get the right PMD information at a instant time, it is necessary to obtain a precise analytical ellipsoid equation to get the three radii $r_{\max }, r_{\operatorname{mid}}, r_{\min }$ and the orientation angles $\alpha, \beta, \gamma$ through data fitting from as little data sampling as possible. In this experiment, we made the data fitting from only 100 data samples using the PSO algorithm, and obtained the DOP ellipsoid shown in Fig. 7. 


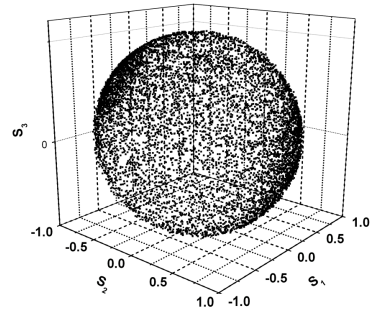

(a) $\mathrm{DGD}=0 \mathrm{ps}$

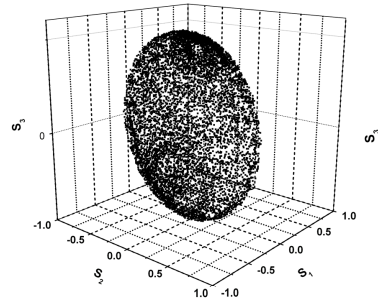

(b) $\mathrm{DGD}=4 \mathrm{ps}$

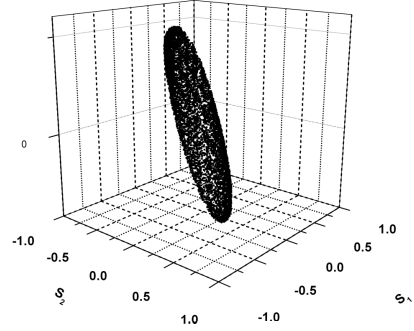

(c) $\mathrm{DGD}=10 \mathrm{ps}$

Fig. 6. 8000 sampling points of output SOPs with various DGD values
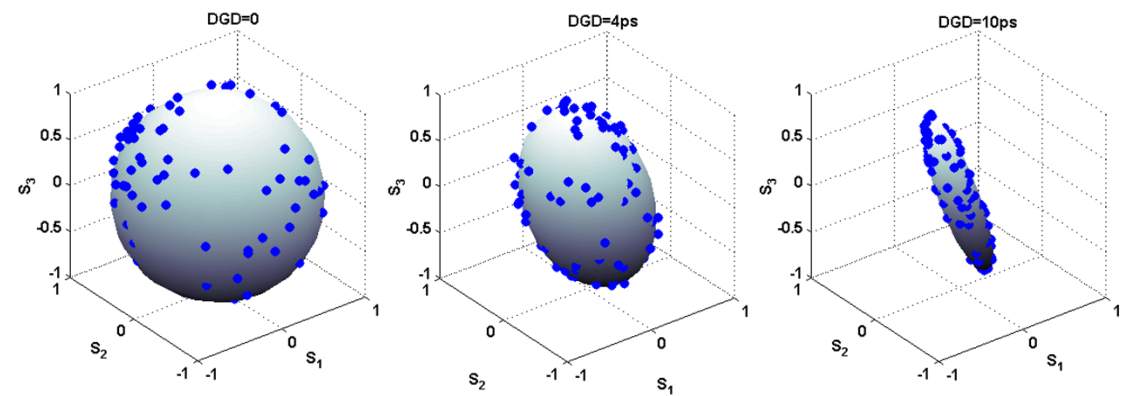

Fig. 7. Data fitting ellipsoid from 100 sampling SOP data using PSO algorithm

Comparing Fig. 6 and Fig. 7, we can see that, the data fitting ellipsoids with 100 samplings nearly fit the ellipsoids formed by 8000 sampling points with high precision. It can be concluded that the PSO algorithm is a powerful one to do the job of data fitting with multi-adjusting parameters, without being trapped in local suboptima. The whole procedure of getting 100 sampling points and ellipsoid data fitting using the PSO algorithm consumed less than 250-ms. The time can be shorter when using higher speed hardware.

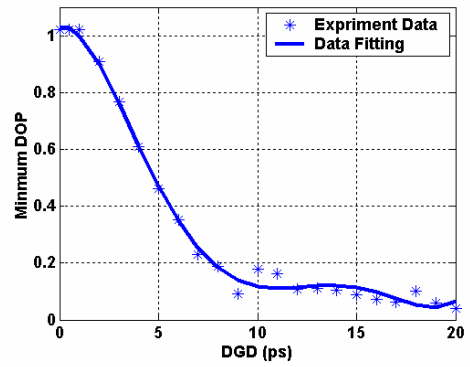

Fig. 8. The length of radius $r_{\min }$ vs. DGD 
We also recorded the relationship between the shortest radius of ellipsoid $r_{\text {min }}$ (minimum DOP) and DGD as shown in Fig. 8. It can be seen that $r_{\text {min }}$ decreases nearly monotonically as DGD increases. So it can be used as the feedback signal for PMD compensation.

\section{Conclusion}

Using the PSO algorithm, we have realized an experiment for determination of the DOP ellipsoid by data fitting using a small number of sampled SOP data. By using the PSO algorithm we can determine the DOP ellipsoid from only 100 samplings with high precision. The 100 data sampling and fitting were achieved within $250 \mathrm{~ms}$. The experiment showed that minimum DOP of the ellipsoid decreased monotonically with DGD, and the direction of ellipsoid orientation was unchanged for the first-order PMD, in accordance with the fact that the PSPs of the PMD vector remain unchanged.

Acknowledgement. This work was supported partly by the National Natural Science Foundation of China (No. 60577046), and Corporative Building Project of Beijing Educational Committee (No. XK100130637).

\section{References}

1. Noé, R., Sandel, D., Yoshida-Dierolf, M., Hinz, S., Mirvoda, V., Schöpflin, A., Glingener, C., Gottwald, E., Scheerer, C., Fischer, G. Weyrauch, T., Haase, W.: Polarization Mode Dispersion Compensation at 10, 20, and $40 \mathrm{~Gb} / \mathrm{s}$ with Various Optical Equaliziers. J. Lightwave Technol. 17 (1999) 1602-1616

2. Rosenfeldt, H., Knothe, Ch., R. Ulrich et al.: Automatic PMD compensation at 40Gbit/s and 80Gbit/s using a 3-dimentional DOP evaluation for feedback. OFC2001, (2001) Postdeadline paper PD27-1

3. Kogelnik, H., Jopson, R. M., Nelson, L.: Polarization-Mode Dispersion, In: Kaminow, I. P., Li, T. (eds): Optical Fiber Telecommunications, IV B. Academic Press, San Diego San Francisco New York Boston London Sydney Tokyo, (2002) 725-861

4. Zhang, X., Yu, L., Zheng, Y., Shen, Y., Zhou, G., Chen, L., Xi, L., Yuan, T., Zhang, J., Yang, B.: Two-Stage Adaptive PMD Compensation in 40Gb/s OTDM Optical Communication System Using PSO Algorithm. Opt. Quantum Electron. 36 (2004) 1089-1104

5. Zheng, Y., Zhang, X., Chen, L., Yang, B.: Analysis of degree of polarization ellipsoid as feedback signal for polarization mode dispersion compensation in NRZ, RZ and CS-RZ systems. Opt. Commun. 234 (2004) 107-117

6. Kennedy, J., Eberhart, R. C.: Paticle Swarm Optimization. In: Proc. of IEEE International Conference on Neural Networks. Piscataway, NJ, USA, (1995) 1942-1948 